\title{
Exploring the effect of biological delays in kinetic models of influenza within a host or cell culture
}

\author{
Benjamin P Holder, Catherine AA Beauchemin
}

\begin{abstract}
Background: For a typical influenza infection in vivo, viral titers over time are characterized by 1-2 days of exponential growth followed by an exponential decay. This simple dynamic can be reproduced by a broad range of mathematical models which makes model selection and the extraction of biologically-relevant infection parameters from experimental data difficult.

Results: We analyze in vitro experimental data from the literature, specifically that of single-cycle viral yield experiments, to narrow the range of realistic models of infection. In particular, we demonstrate the viability of using a normal or lognormal distribution for the time a cell spends in a given infection state (e.g., the time spent by a newly infected cell in the latent state before it begins to produce virus), while exposing the shortcomings of ordinary differential equation models which implicitly utilize exponential distributions and delay-differential equation models with fixed-length delays.
\end{abstract}

Conclusions: By fitting published viral titer data from challenge experiments in human volunteers, we show that alternative models can lead to different estimates of the key infection parameters.

\section{Background}

In the past decade, mathematical models of viral infection have been successfully applied to a number of problems on the periphery of the annual public health problem that is influenza [1]. In the laboratory, mathematical models have aided the development of efficient vaccine production techniques [2] and improved the quantitative characterization of antiviral drug action [3]. Mathematical models have also improved our understanding of the course of the disease within human [4] and animal hosts [5]. Because these models serve as a bridge between the microscopic scale (where virus interacts with cell) and the macroscopic scale (where the infection is manifested as a disease) they will inevitably be applied in the future to pressing public health questions such as the estimation of virulence and fitness for emerging strains, the spread of drug resistance and, more generally, the connections between viral genotypic information and clinical data.

The success of a within-host virus infection model depends on an accurate representation of biological reality. This allows a model not only to describe the phenomenon

\footnotetext{
* Correspondence: cbeau@ryerson.ca

Department of Physics, Ryerson University, Toronto, ON, M5B 2K3, Canada Full list of author information is available at the end of the article
}

under consideration, but also to make reliable predictions about unobserved consequences. For example, in 1995 a simple model of HIV dynamics was applied to describe the observed exponential clearance of virus under the administration of a drug suppressing viral production [6]. The primary result of this work, however, was not the description of viral clearance itself, but the prediction of dynamics in the absence of drug, i.e., that high viral clearance must be balanced by high viral production, which in turn allows for extremely rapid mutation of the virus strain. This conclusion had important implications for the development of therapy, specifically the necessity of a "drug cocktail". For influenza infections, the primary clinical data available to a mathematical modeler is the viral titer over the course of an infection, usually obtained by a daily nasal wash collected from an infected patient. This data generally follows a simple functional form in time which can be reproduced by a variety of dynamical models. Thus, if meaningful information is to be extracted from such data, the model applied must already be a trusted simulator of the underlying infection kinetics. In this paper, we consider evidence from laboratory infection experiments which must inform the construction of a mathematical model, focusing specifically on the 
implementation of the time spent by a cell in each of the various stages of infection.

The basic viral infection model $[4,7]$ assumes interaction of virus with cells in four different states (Figure 1), and is based on a coarse-grained view of the virus replication cycle. Cells that have not yet been infected by the virus, but are susceptible to infection, are considered target cells $(T)$. The interaction of virus with target cells leads to these cells becoming latently $(L)$ infected (i.e., infected but not producing virus). After infection, a time, $t_{L}$, passes - as the virus particle is unpacked, its genome is delivered to the cell nucleus, replication begins, and new particles assemble at the plasma membrane - before new virus particles are released and the cell enters the infectious (I) state. After a subsequent time, $t_{I}$, the infectious cell halts virus production and transitions into a state we will refer to as dead $(D)$.

The implementation of a particular dynamical structure on this basic model requires a more detailed specification of the biological processes. The infection of cells (the transition of target cells to latently-infected cells) has been observed to be a Poisson process where the rate of infection is proportional to the local virus concentration [8] and it is implemented in the model as a continuous representation of that stochastic process. Virus production by infectious cells can be assumed to proceed at a constant rate and the infectivity of free virus is known to decrease exponentially in time [3,9], leading to a simple equation for virus dynamics. To complete the dynamical description, one must specify how a latently infected cell becomes infectious and for how long infectious cells produce virus. In other words, one must specify the distribution of the delays, $t_{L}$ and $t_{I}$, between the states of infection.

In an epidemiological context, the problem of implementing generic delays between infected classes was first considered by Kermack and McKendrick in their seminal 1927 work on infectious disease dynamics [10].
Hethcote and Tudor [11] introduced a general approach to the problem, using a probability density function for the time spent in a given state, which has been applied frequently in the field of mathematical epidemiology (see, e.g., [12-14] and references therein). Here, we will apply the same approach to within-host influenza viral infections, resulting in a model with differential equations to describe target cell and virus dynamics, and integral equations to describe the latent and infectious cell populations (a similar approach was considered for HIV in [15]).

Mathematically, the simplest choice of delay distribution is exponential (shown in Figure 2 with a few other choices), because it reduces the model to a system of ordinary differential equations (ODEs). For that reason, it is the most commonly-used model type for both epidemiological and within-host problems. In viral infections, however, the assumption of an exponential distribution seemingly conflicts with the biological evidence. For example, if the time of latent infection is chosen from such a distribution, the model would predict that a significant fraction of cells begin producing virus almost immediately after infection. In reality, however, there is always a minimum delay prior to viral release: endocytosis and the fusion of the viral envelope with the endosome takes, on average, half an hour [16]; the viral RNA enters the nucleus in most cells within the next one hour [17]; mRNA is transcribed in the nucleus, then transported back to the cytoplasm for translation and newly formed M1 matrix proteins are observed only three hours after infection, on average, and hemagglutinin four hours post-infection [17]; newly formed glycoproteins, matrix proteins and nucleocapsids then must assemble at the cell membrane, bud off and be cleaved from the sialic acid receptors [18]. Each of these steps and their timings depend on virus strain and cell type, and one can expect significant variation between cells, but a long delay without viral production

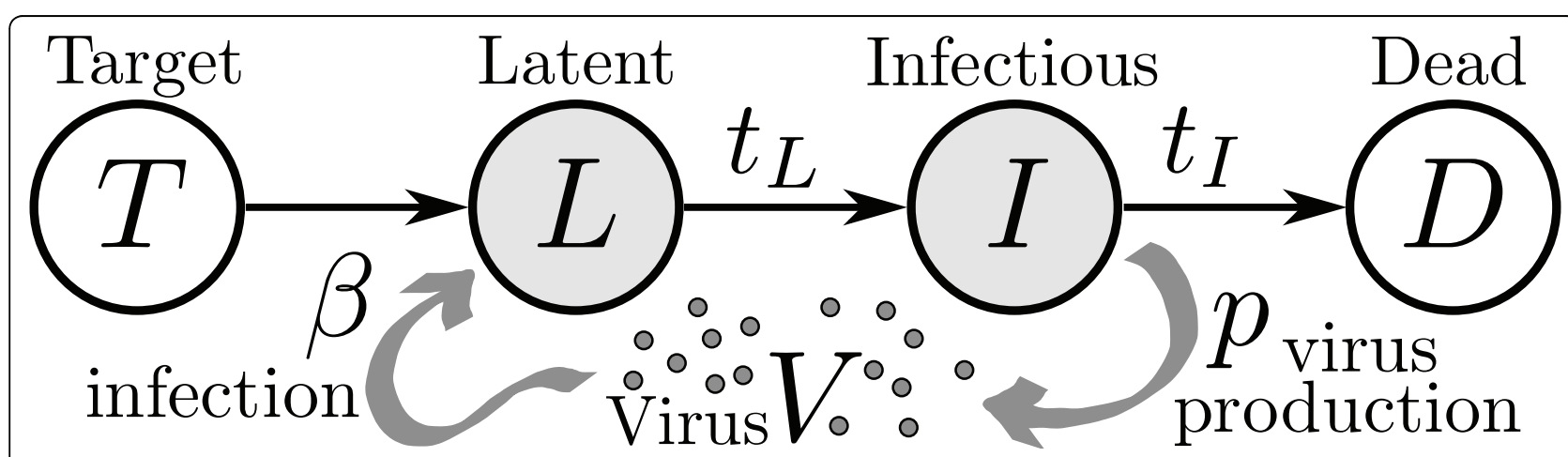

Figure 1 Basic viral infection model. The cell-virus interactions of infection $(\boldsymbol{\beta})$ and viral production $(p)$ are indicated along with the four possible states in which cells can be found. The lifetimes of the latent and infectious states for a particular cell, $t_{L}$ and $t_{l}$, are considered random variables. 


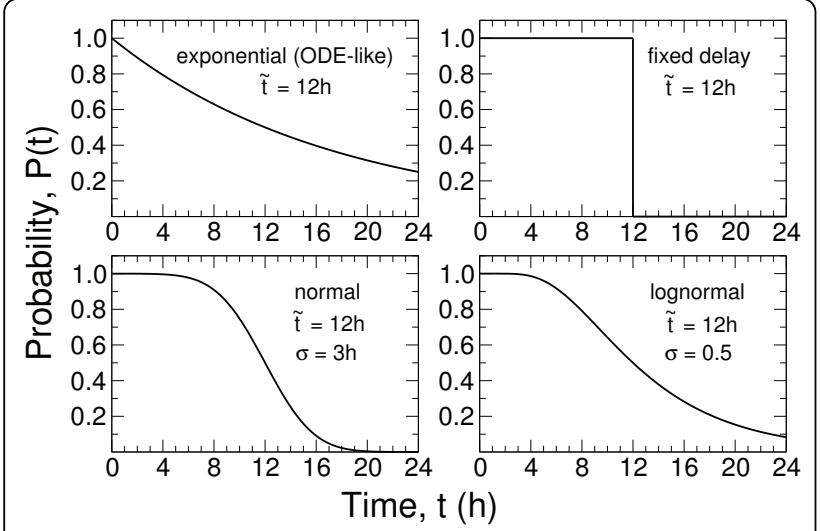

Figure 2 Example delay distributions. Four example delay types defining the time spent by a cell in a given state. The plotted function, $P(t)$ corresponds to the probability that after some time $t$, the cell remains in its current state, i.e., has not yet transitioned to the next state. In each example, $\tilde{t}=12 \mathrm{~h}$ is the median time of transition to the next state. While exponential-delays allow for instantaneous transitions (e.g., 10\% of cells will have transitioned after $1.5 \mathrm{~h}$ has elapsed), the other three types enforce a minimum waiting period for almost all cells before transitioning from one state to the other (i.e., $P(t) \approx 1$ for $0<t<t_{\text {min }}$ ). Enforcing this delay is more biologically realistic. The function $P(t)$ appears explicitly in the viral infection model, Equation (1), and allows for generic delays between states of infection.

is an essential characteristic of the infection cycle. Influenza virus-induced cell death is less well characterized: the mechanism of cell killing (apoptosis or necrosis) depends on cell type $[19,20]$, and the timing of apoptosis in particular is strongly strain dependent [21]. In this situation, a broad freedom in selecting the distribution for infectious cell lifespans is warranted.

Despite questions about their biological appropriateness, ODE models have had success in describing in vivo infection data (for influenza see, for example, $[4,5])$. Models with non-exponential delays have been similarly successful, including those with Dirac delta function transition distributions, leading to a delay-differential model $[3,4,22]$; and multi-compartmental ODE models (with $n$ sequential phases of infection) yielding delays with a gamma-function distribution [23-25] Here, we consider a set of in vitro experiments which allows for some discrimination between models, namely the single-cycle viral yield assay. By fitting models with different transition distributions (Figure 2) to single-cycle assay data, we show that the correct implementation of delays is crucial to the success of a model in describing these assays. Using these results, we consider in vivo data from challenge experiments in humans to explore how the choice of delays affects the parameter values extracted when fitting the model to experimental data.

\section{Methods}

Model

The general viral infection model used in this paper is written, following [11], as

$$
\begin{aligned}
\dot{T} & =-\beta T V \\
L(t) & =L(0) P_{L}(t)+\int_{0}^{t} \beta T(s) V(s) P_{L}(t-s) d s \\
I(t)= & I(0) P_{I}(t)+\int_{0}^{t} P_{I}(t-s) L_{(0)} f_{L}(s) d s \\
& +\int_{s_{2}=0}^{t} \int_{s_{1}=0}^{s_{2}} \beta T\left(s_{1}\right) V\left(s_{1}\right) \cdot f_{L}\left(s_{2}-s_{1}\right) \cdot P_{I}\left(t-s_{2}\right) d s_{1} d s_{2} \\
\dot{V}= & p \frac{I}{N}-c V
\end{aligned}
$$

where $T, L, I$ are the populations of cells in the target, latently-infected and infectious (virus-producing) states, respectively, and $N$ the total number of cells in the system. $V$ is the virus concentration, $\beta$ and $p$ are the rates of infection and virus production, respectively, and $c$ is the viral clearance rate. The function $P_{L}(t)$ is the probability that a cell remains in the latent state for at least a time $t$ before transitioning to the infectious state, and $P_{I}(t)$ is the probability that a cell remains in the infectious state for at least a time $t$ before transitioning to the dead state (i.e., before it ceases to release virus). The transition profiles for different choices for the expression of $P(t)$ are illustrated in Figure 2. $f_{L}$ is the probability density function for the time a cell will spend in the latent state before transitioning to the infectious state $\left(f_{L}=-\mathrm{d} P_{L} / \mathrm{d} t\right)$. Note that $f_{I}(t)$ does not explicitly appear in the model.

\section{Delay distributions}

If an exponential distribution is chosen for both transitions (exponential-delay), we have

$$
f_{L}(t)=\frac{1}{\tau_{L}} \exp \left[-\frac{t}{\tau_{L}}\right], P_{L}(t)=\exp \left[-\frac{t}{\tau_{L}}\right], \text { and } P_{I}(t)=\exp \left[-\frac{t}{\tau_{I}}\right]
$$

where $\tau_{L}$ is the average time spent by a cell in the latent infection state, and $\tau_{I}$ is the average lifespan of an infectious cell. Equations (1a-1d) can then be written as a system of ordinary differential equations

$$
\begin{aligned}
& \dot{T}=-\beta T V \\
& \dot{L}=\beta T V-\frac{L}{\tau_{L}} \\
& \dot{I}=\frac{L}{\tau_{L}}-\frac{I}{\tau_{I}} \\
& \dot{V}=p \frac{I}{N}-c V .
\end{aligned}
$$

When a Dirac delta function is used for both $f_{L}$ and $f_{I}$ (fixed-delay), such that the times spent by cells in the 
latent state and the infectious state are exactly $\tau_{L}$ and $\tau_{I}$, respectively, Equations (1a-1d) reduce to a set of delay differential equations (DDE) [3].

Biologically, cells transition from one state to another only after some average time has elapsed. One also expects to see some variation in these transition times among cells as many of the processes rely on chains of stochastic intracellular events. Thus, normal distributions,

$$
\begin{gathered}
f_{L}(t)=\frac{A_{L}}{\sqrt{2 \pi \sigma_{L}^{2}}} \exp \left[-\frac{\left(t-\tau_{L}\right)^{2}}{2 \sigma_{L}^{2}}\right], P_{L}(t)=1-\frac{A_{L}}{2}\left[\operatorname{erf}\left(\frac{t-\tau_{L}}{\sqrt{2 \sigma_{L}^{2}}}\right)+\operatorname{erf}\left(\frac{\tau_{L}}{\sqrt{2 \sigma_{L}^{2}}}\right)\right], \\
\quad \text { and } P_{I}(t)=1-\frac{A_{I}}{2}\left[\operatorname{erf}\left(\frac{t-\tau_{I}}{\sqrt{2 \sigma_{I}^{2}}}\right)+\operatorname{erf}\left(\frac{\tau_{I}}{\sqrt{2 \sigma_{I}^{2}}}\right)\right],
\end{gathered}
$$

where $\sigma_{L}$ and $\sigma_{I}$ are the standard deviations, are a natural choice (normal-delay). The normalization constants, $A_{i}$, are necessary since the distributions must be truncated at zero. If one wishes to avoid renormalization, a good alternative is a lognormal distribution (lognormaldelay)

$$
\begin{aligned}
& f_{L}(t)=\frac{1}{t \sqrt{2 \pi \sigma_{L}^{2}}} \exp \left[-\frac{\left(\ln t-\ln \tau_{L}\right)^{2}}{2 \sigma_{L}^{2}}\right], P_{L}(t)=\frac{1}{2} \operatorname{erfc}\left(\frac{\ln t-\ln \tau_{L}}{\sqrt{2 \sigma_{L}^{2}}}\right) \\
& \quad \text { and } P_{I}(t)=\frac{1}{2} \operatorname{erfc}\left(\frac{\ln t-\ln \tau_{I}}{\sqrt{2 \sigma_{I}^{2}}}\right)
\end{aligned}
$$

where $\operatorname{erfc}(x)$ is the complementary error function (i.e., $\operatorname{erfc}(x)=1-\operatorname{erf}(x))$ and the standard deviations $\sigma_{L}$ and $\sigma_{I}$ are dimensionless quantities.

To facilitate comparison between the various distributions, we utilize their median values. For the Dirac delta and lognormal distributions, the median is simply the value of the parameter itself, i.e.,

$$
\tilde{t}_{L}=\tau_{L} \text { and } \tilde{t}_{I}=\tau_{I} \text { (fixed and lognormal). }
$$

For exponential distributions, the median is

$$
\tilde{t}_{L}=\tau_{L} \ln 2 \text { and } \tilde{t}_{I}=\tau_{I} \ln 2 \text { (exponential), }
$$

and for the truncated normal, the median is found by setting its cumulative distribution function to one-half (there is no simple analytical expression).

\section{Numerical simulation}

Numerical evaluation of the model in Equation (1) was performed using a modified Euler technique. At every time step of length $\Delta t$, newly infected cells, $L_{\text {new }}=\beta T V$ $\Delta t$, were removed from the target population. The passage of these $L_{\text {new }}$ cells through the latent and infectious states was then calculated for all future times using $f_{L}$, $P_{L}$ and $P_{I}$ and added to that of previously transitioned cells. Virus dynamics at each time step were calculated according to the Euler approximation of Equation (1d). Simulations of single-cycle in vitro experiments were initialized with $L(0)=N$; simulations of in vivo infections were initialized with $T(0)=N$ and $V(0)=V_{0}$.

\section{Model fitting and parameter extraction}

In any model-fitting exercise, a number of considerations must be made to ensure the reliable extraction of parameter values from experimental data. First, one must consider the question of parameter identifiability $[36,57,58]$ : If the experimental system were to exactly reproduce the dynamics of the model equations, could the parameters be uniquely identified from the available observations? A number of techniques have been introduced to address identifiability for ODE models [37,59], but these are not directly applicable to the more general system considered here (Equation (1)). Nevertheless, for each experiment considered here we attempted to reduce consideration to an identifiable set of parameters. For the early phase single-cycle viral yield experiments, we fixed parameter values involving viral clearance and infectious cell death, and fitted only those parameters related to viral production and the transition of cells from the latent to infectious state. In the single-cycle, single-history experiment, independent information on viral clearance allowed for that parameter to be fixed in fitting. For the in vivo volunteer patient infection data, we have arbitrarily fixed the product of the viral production and infection rates in order to obtain a unique solution in the fitting procedure.

A second consideration when fitting experimental data is the question of model error: Is the mathematical model an appropriate representation of experimental data? To address this question, we performed least squares fitting of the model equations to the data sets using the Octave 3.2.4 [60] implementation of the Levenberg-Marquardt algorithm, leasqr. For all data presented here, fits were performed to the viral titer data in log-space and the sum of squared residuals (SSR) was calculated as $\sum_{i}\left(\log V_{i}^{\text {data }}-\log V_{i}^{\text {model }}\right)^{2}$.

In order to compare model systems with different numbers of parameters, we evaluated the Akaike information criterion (AIC) for each fit,

$$
\mathrm{AIC}_{\mathrm{c}}=\mathrm{AIC}+\frac{2 k(k+1)}{n-k-1}=2 k+n \ln \left(\frac{\mathrm{SSR}}{n}\right)+\frac{2 k(k+1)}{n-k-1}
$$

where $k$ is the number of model parameters, $n$ is the number of data points and $\mathrm{AIC}_{\mathrm{c}}$ is the "corrected" form of the AIC for small sample sizes [61].

Finally, to account for measurement error, we calculated $95 \%$ confidence intervals for each reliably extracted parameter value by fitting 1000 bootstrap replicates [62] Confidence intervals were not calculated for models fits 
with large error (high SSR and $\mathrm{AIC}_{\mathrm{c}}$ ) or for fits to volunteer patient infection data, where arbitrary assumptions were made to constrain the fitted parameter values.

\section{Calculation of the basic reproductive number}

For each fit to the volunteer patient infection data we numerically calculated an approximation of the basic reproductive number, $R_{0}$. In the ODE model, Equation (3), linear stability of the disease-free equilibrium is guaranteed by $R_{0}<1$, where

$$
R_{0}=\frac{p \beta}{c \delta}
$$

It can also be shown using the ODE that this quantity is equal to the commonly-quoted definition of the reproductive number: the number of secondary infections caused by one infectious cell, in a completely susceptible cell population. For other delay models, where an analytical form is not readily available, we calculated $R_{0}$ numerically according to that statement, i.e., for a given set of parameters, we disallowed latent to infectious transitions, initialized the simulation with one infectious cell, and determined the number of cells in the latent state as $t \rightarrow \infty$.

\section{Results and discussion}

\section{General features of single-cycle viral growth}

Single-cycle growth (SCG) viral yield experiments provide a unique view of viral replication. By initiating infection with a viral inoculum of high concentration (a multiplicity of infection (MOI) much larger than one), all cells are infected simultaneously, and the experimentalist effectively synchronizes the cells' passage through the phases of latency, viral production and death. The resulting viral production curve can then be viewed as that of the average cell. This is in sharp contrast to "multiple-cycle" yield experiments $(\mathrm{MOI} \ll 1)$, where only a few cells are initially infected, leading to successive cycles of infection; the resulting exponential growth of both infected cells and virus over time effectively masks the dynamics of a single cell. Virus infections of humans and animals are similar to this latter experiment in that they are likely initiated by the infection of a only a few cells $[26,27]$, leading to the exponential consumption of a large target cell population. Thus, SCG experiments demonstrate an artificial infection dynamic which would never occur in nature. However, their depiction of the average virus production of a cell makes them an invaluable tool for model building and for isolating specific components or parameters of the viral replication cycle.

In the original publications of the nine example SCG data sets plotted in Figure 3 [9,28-32] virus was plotted on a logarithmic scale, due to the extraordinary sensitivity of measurement techniques which can detect infectious virus over nearly 8 orders of magnitude. It is clear from Figure 3, however, that this perspective masks the most important features of SCG: a long delay without significant viral release followed by linear growth of the virus concentration. This simple dynamic can be summarized in the empirical expression,

$$
V(t)=\left\{\begin{array}{cc}
\left(t-\tau_{L}^{*}\right) p^{*} & \left(t \geq \tau_{L}^{*}\right) \\
0 & \text { otherwise }
\end{array}\right.
$$

where $\tau_{L}^{*}$ and $p^{*}$ can be considered approximations of the average latent infection period, $\tau_{L}$, and the viral production rate, $p$, in the case where viral clearance is low (i.e., Equation (8) is the solution of Equation (1d) when $I / N=1$ and $c=0)$. The fitted values of $\tau_{L}^{*}$ and $p^{*}$ for all nine experiments are given in Table 1 .

We will consider below the characterization of the SCG experiment using various dynamical models, but some simple analysis can be done using only the empirical relation above. For example, two experiments (Figure $3 \mathrm{~A}-\mathrm{B}$ and Figure $3 \mathrm{C}-\mathrm{D})$ considered the growth of influenza A/Udorn/307/72 (H3N2) and a counterpart strain possessing a single mutation in the NS1 gene (T215A and R83A, respectively) $[28,29]$. In each case, the experiment reveals a significant reduction of the approximate viral production rate $p^{*}$ for the mutant, without a significant change in the approximate latency period. This shows that the single-cycle experiment can highlight important biological characteristics of a virus strain, with very little mathematical analysis.

\section{Characterizing the latent infection period from single- cycle growth assays}

To determine the type of distribution which should be used to model the time spent by a newly infected cell in the latent phase, we performed model fits to two singlecycle data sets $[28,32]$ which offered frequent sampling in time and a large range of virus measurements (Figure 4). Recently we have shown [33] that the ODE assumption of exponential delays yields a very poor fit to single-cycle viral yield data. Here, we considered three additional distributions for the waiting times: fixed-delay, normaldelay and lognormal-delay. We neglected the influence of infectious cell death by setting the infectious cell lifespan to be much longer than the duration of the experiment. This is an oversimplification of reality, particularly for later times. However, the lack of data spanning the period of virus decline, which occurs at later times than those measured, prohibits any real measure of infectious cell death. Similarly, this lack of viral decay information prohibits an effective characterization of the loss of virus 


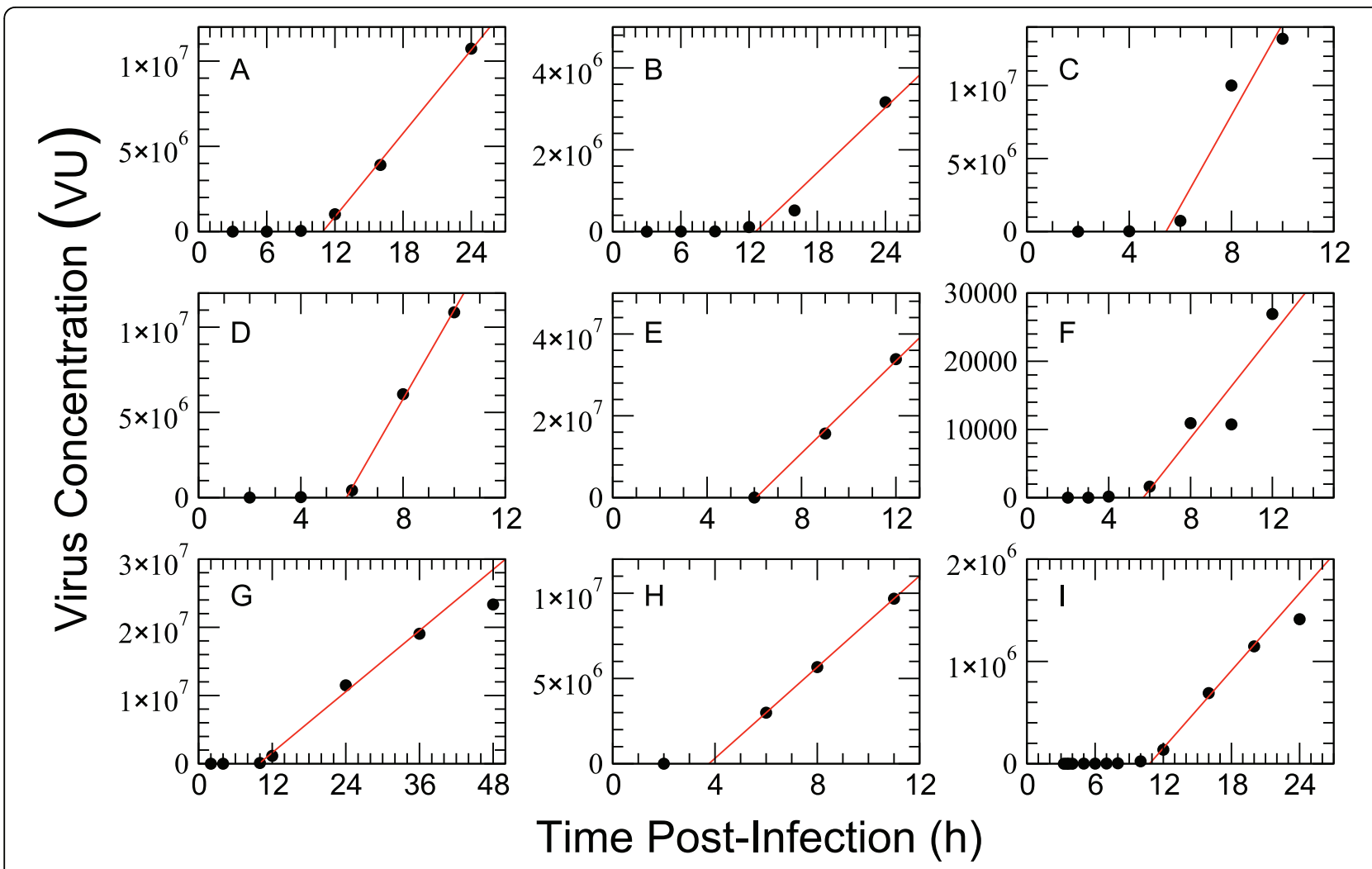

Figure 3 Single-cycle viral yield examples. Examples of single-cycle growth experiments. Plotted lines are fits to the non-zero data points using Equation 8 (the last data point in $\mathbf{G}$ and $\mathbf{I}$ have been excluded from the fits) Virus units (VU), the parameters of the fit, and the details of each experiment are given in Table 1.

infectivity. Therefore, we fitted the model using two values of viral clearance, zero and $0.2 \mathrm{~h}^{-1}$. The latter value represents an approximate upper-bound of the viral clearance value based on literature reports for in vitro experiments performed at $37^{\circ} \mathrm{C}[2,3]$.

The results of model fits to the two data sets are collected in Table 2. The exponential- and fixed-delay models clearly provide a poorer fit to the data than those with normal- and lognormal-delays (for the A/ Udorn/307/72 [28] data set, however, the small number of data points and fewer parameters, with respect to normal and lognormal models, do not allow for their formal exclusion based on $\mathrm{AIC}_{\mathrm{c}}$ ). While the exponential-delay model (ODE) is unable to duplicate any

Table 1 Information on the individual single-cycle viral yield experiments plotted in Figure 3

\begin{tabular}{|c|c|c|c|c|c|c|}
\hline (Label) Strain & Cell Type & $\mathrm{MOI}^{\mathrm{a}}$ & $\mathrm{Vu}^{\mathrm{b}}$ & $p^{*}(\mathrm{VU} / \mathrm{h})$ & $\tau_{L}^{*}(\mathbf{h})$ & ref. \\
\hline (A) A/Udorn/307/72 (H3N2) & A549 & 3 & $\mathrm{PFU} / \mathrm{mL}$ & $(8.2 \pm 0.3) \times 10^{5}$ & $10.9 \pm 0.8$ & [28] \\
\hline (B) $\mathrm{A} /$ Udorn/307/72 $(\mathrm{H} 3 \mathrm{~N} 2)^{\mathrm{C}}$ & A549 & 3 & $\mathrm{PFU} / \mathrm{mL}$ & $(2.7 \pm 0.6) \times 10^{5}$ & $12 \pm 5$ & [28] \\
\hline (C) A/Udorn/307/72 (H3N2) & A549 & 5 & $\mathrm{PFU} / \mathrm{mL}$ & $(3.1 \pm 0.9) \times 10^{6}$ & $(6 \pm 3)$ & [29] \\
\hline (D) A/Udorn/307/72 (H3N2) & A549 & 5 & $\mathrm{PFU} / \mathrm{mL}$ & $(2.6 \pm 0.1) \times 10^{6}$ & $5.8 \pm 0.4$ & [29] \\
\hline (E) A/Udorn/307/72 (H3N2) & MDCK & 5 & $\mathrm{PFU} / \mathrm{mL}$ & $(5.6 \pm 0.3) \times 10^{6}$ & $(6.1 \pm 0.4)$ & [29] \\
\hline (F) A/PR/8/34 (H1N1) & MDCK & 10 & $\mathrm{TCID}_{50} / \mathrm{mL}$ & $(4 \pm 1) \times 10^{3}$ & $6 \pm 3$ & [30] \\
\hline (G) $\mathrm{A} / \mathrm{PR} / 8 / 34$ (H1N1) & MDCK & 32 & PFU & $(7.5 \pm 0.4) \times 10^{5}$ & $(9.8 \pm 1)$ & {$[9]$} \\
\hline (H) $\mathrm{A} / \mathrm{X}-31(\mathrm{H} 3 \mathrm{~N} 2)$ & Vera & 50 & $\mathrm{TCID}_{50} / \mathrm{mL}$ & $(1.34 \pm 0.01) \times 10^{6}$ & $(3.75 \pm 0.01)$ & [31] \\
\hline (I) A/NWS/33 (H1N1) & $1-5 C-4$ & 50 & $\mathrm{PFU} / \mathrm{mL}$ & $(1.26 \pm 0.07) \times 10^{5}$ & $10.8 \pm 1$ & [32] \\
\hline
\end{tabular}

a - The number of cells used in each experiment was $\sim 2 \times 10^{6}$ except for (F) and (G) which had $9 \times 10^{6}$ and $3 \times 10^{5}$ cells, respectively.

b - Virus Unit. It should be noted that this unit is inherently experiment-specific, depending on virus strain, cell type and experimental details. Quantities which depend on VU (e.g., $p^{*}$ ) cannot be directly compared between experimental groups.

$c-N S 1-T 215 A$ recombinant mutant.

d - NS1-R38A recombinant mutant. 


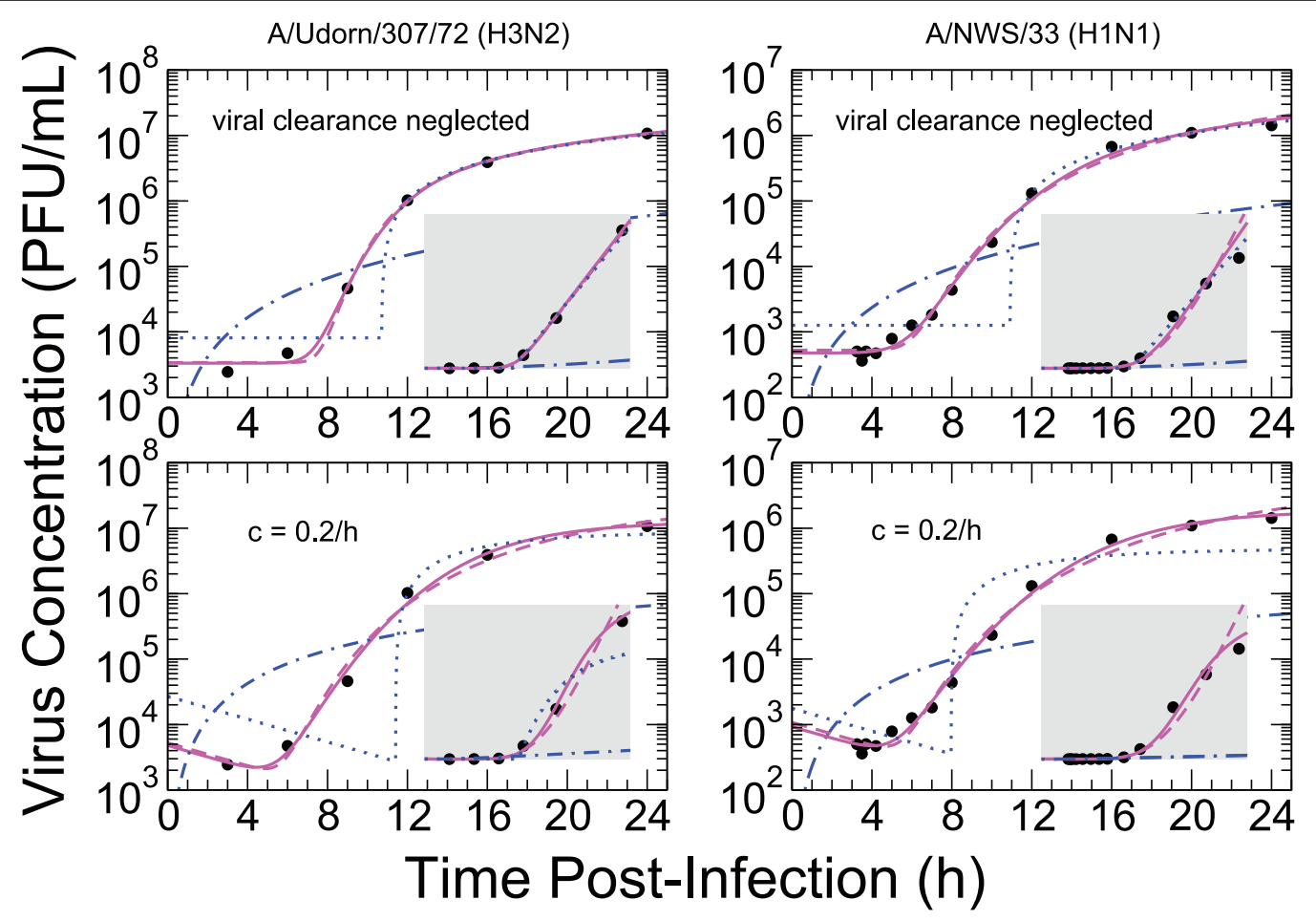

Figure 4 Model fits to viral titers from single-cycle experiments. Model fits to viral titers from single-cycle experiments by (Left) Hale et. al. [28] (Figure 3A) and (Right) Sugiura and Kilbourne [32] (Figure 31). Fitting was performed using an exponential (dot-dashed), delta (dotted), normal (solid) or lognormal (dashed) distribution for the time spent by newly infected cells in the latent state before transitioning to the infectious state. Insets show the same fits and data on a linear virus scale (axis ranges are identical to those in Figure 3).

feature of the dynamics, the fixed-delay model fits the data well at late times in the infection when the dynamics are dominated by linear growth, at least when viral clearance is neglected. It does a poor job, however, of describing the early stages of virus release, and is unable to provide the correct dynamics in the presence of viral clearance.

Both the normal- and lognormal-delay models provide an adequate description of the data over the entire range of values and for both values of viral clearance, although the SSR and $\mathrm{AIC}_{\mathrm{c}}$ values are smaller for fits using a normal distribution. When the fits to the logvalued virus are viewed in linear-space (inset graphs), the normal fits appear to be a more reasonable approximation of the data in that the linear SSRs of these fits, which depend most sensitively on the larger virus values, are also smaller. When viral clearance is neglected, the fitted values of the viral production rate, $p$, are close to the approximate values of $p^{*}$ (Table 1 ). Non-zero viral clearance leads to a larger fitted production rate, as expected. The fitted values for the median latent infection period, $\tilde{t}_{L}$, vary depending on the distribution type, but are always as long as the approximate values of $\tau_{L}^{*}$ for both experiments (10.9 $\mathrm{h}$ for [28] and $10.8 \mathrm{~h}$ for [32]). The introduction of a non-zero viral clearance leads to even longer latent infection periods, ranging from $8 \mathrm{~h}$ to $24 \mathrm{~h}$. The fitted standard deviations, $\sigma_{L}$, are between 1.5 and $3.0 \mathrm{~h}$ for the normal distribution and between 0.15 and 0.44 for the lognormal distribution.

\section{Characterizing the infection cycle from a single-cycle, single-history yield assay}

In 1968, an in vitro experiment was performed which, to our knowledge, is unique in the literature [9]. Like the experiments presented in the previous sections, a SCG experiment was prepared: $\sim 10^{7}$ cells were incubated with a high titer $(\mathrm{MOI}=10)$ of influenza $\mathrm{A} / \mathrm{PR} / 8$ (H1N1) virus such that almost all cells were infected and then the infection medium was removed. Unlike typical SCG experiments, however, viral titer was not measured by sacrificing independent wells at each sampling time to titrate their overlay. Instead, the liquid overlay from the same well was removed in its entirety and replaced with fresh, virus-free medium and the infection was allowed to continue. Thus, titrations of the collected overlay medium provided a measure of the amount of virus being produced by a single cell culture at the time of collection. Application of this sampling protocol to SCG experiments - which we refer to as a 
Table 2 Fits to two single-cycle viral yield experiments (Figure 4)

\begin{tabular}{|c|c|c|c|c|c|c|c|c|}
\hline \multicolumn{9}{|c|}{ A/Udorn/307/72 (H3N2) [28] } \\
\hline Dist $^{\mathrm{a}}$ & $c\left(\mathrm{~h}^{-1}\right)$ & $V_{0}\left(\frac{\mathrm{PFU}}{\mathrm{mL}}\right)$ & $p\left(\frac{\mathrm{PFU}}{\mathrm{mL} \cdot \mathrm{h}}\right)$ & $\tau_{L}(\mathrm{~h})$ & $\sigma_{L}$ & $\tilde{t}_{L} \quad$ (h) & SSR & $\mathrm{AIC}_{\mathrm{c}}$ \\
\hline $\exp$ & $(0)^{\mathrm{b}}$ & 0 & $2.4 \times 10^{6}$ & 1200 & - & 830 & 4.86 & 16.7 \\
\hline$\delta$ & $(0)$ & $8.1 \times 10^{3}$ & $7.7 \times 10^{5}$ & 10.7 & - & 10.7 & 0.90 & 6.6 \\
\hline \multirow[t]{2}{*}{ N } & (0) & $3.3 \times 10^{3}$ & $8.3 \times 10^{5}$ & 11.1 & $1.5 \mathrm{~h}$ & 11.1 & 0.039 & 17.8 \\
\hline & & {$[2.5: 4.4]^{c}$} & {$[5.9: 12.7]$} & {$[9.8: 12.5]$} & {$[0.8: 2.2]$} & & & \\
\hline \multirow[t]{2}{*}{$\ln N$} & (0) & $3.4 \times 10^{3}$ & $8.2 \times 10^{5}$ & 10.9 & 0.15 & 10.9 & 0.041 & 18.1 \\
\hline & & {$[2.4: 4.1]$} & {$[5.6: 12.8]$} & {$[9.7: 12.6]$} & {$[0.08: 0.22]$} & & & \\
\hline $\exp$ & $(0.2)$ & 0 & $10^{36}$ & $10^{32}$ & - & $10^{32}$ & 5.79 & 17.8 \\
\hline$\delta$ & $(0.2)$ & 27000 & $1.8 \times 10^{6}$ & 11.4 & - & 11.4 & 1.73 & 10.5 \\
\hline \multirow[t]{2}{*}{$N$} & $(0.2)$ & $4.8 \times 10^{3}$ & $2.6 \times 10^{6}$ & 14.0 & $2.7 \mathrm{~h}$ & 14.0 & 0.068 & 21.1 \\
\hline & & {$[3.1: 7.7]$} & {$[1.8: 4.4]$} & [13.0:15.9] & {$[2.3: 3.3]$} & & & \\
\hline \multirow[t]{2}{*}{$\ln N$} & $(0.2)$ & $5.3 \times 10^{3}$ & $5.2 \times 10^{6}$ & 19.3 & 0.37 & 19.3 & 0.144 & 25.6 \\
\hline & & {$[2.8: 10]$} & {$[1.7: 11.0]$} & [12.5:57.9] & {$[0.22: 0.58]$} & & & \\
\hline \multicolumn{9}{|c|}{ A/NWS/33 (H1N1) [32] } \\
\hline Dist & $c\left(h^{-1}\right)$ & $V_{0}\left(\frac{\mathrm{PFU}}{\mathrm{mL}}\right)$ & $p\left(\frac{\mathrm{PFU}}{\mathrm{mL} \cdot \mathrm{h}}\right)$ & $\tau_{\llcorner}(\mathrm{h})$ & $\sigma_{\llcorner}$ & $\tilde{t}_{L}$ & SSR & $\mathrm{AlC}_{\mathrm{c}}$ \\
\hline $\exp$ & (0) & 0 & $1.4 \times 10^{26}$ & $4.8 \times 10^{23}$ & - & $3.3 \times 10^{23}$ & 8.30 & 2.8 \\
\hline$\delta$ & (0) & 1300 & $1.2 \times 10^{5}$ & 10.9 & - & 10.9 & 2.77 & -11.4 \\
\hline \multirow[t]{2}{*}{ N } & (0) & $4.7 \times 10^{2}$ & $1.5 \times 10^{5}$ & 12.7 & $2.5 \mathrm{~h}$ & 12.7 & 0.090 & -51.7 \\
\hline & & {$[3.8: 5.7]$} & {$[1.1: 2.2]$} & {$[11.5: 14.1]$} & {$[2.1: 3.0]$} & & & \\
\hline \multirow[t]{2}{*}{$\ln N$} & (0) & $5.3 \times 10^{2}$ & $2.3 \times 10^{5}$ & 15.3 & 0.33 & 15.3 & 0.167 & -43.6 \\
\hline & & {$[4.1: 6.6]$} & {$[1.1: 9.2]$} & [11.6:26.1] & {$[0.24: 0.47]$} & & & \\
\hline $\exp$ & (0) & 0 & $4.5 \times 10^{31}$ & $9.1 \times 10^{28}$ & - & $6.3 \times 10^{28}$ & 11.0 & 6.5 \\
\hline$\delta$ & $(0.2)$ & $1.8 \times 10^{3}$ & $9.6 \times 10^{4}$ & 7.9 & - & 7.9 & 2.11 & -15.0 \\
\hline \multirow[t]{2}{*}{$N$} & $(0.2)$ & $9.3 \times 10^{2}$ & $3.8 \times 10^{5}$ & 14.4 & $3.0 \mathrm{~h}$ & 14.4 & 0.099 & -50.4 \\
\hline & & [3.9:11] & [1.1:5.9] & [11.6: 21.9] & {$[0.3: 3.3]$} & & & \\
\hline \multirow[t]{2}{*}{$\ln N$} & $(0.2)$ & $1.1 \times 10^{3}$ & $1.2 \times 10^{6}$ & 23.8 & 0.44 & 23.8 & 0.22 & -40.0 \\
\hline & & [0.79:1.4] & {$[0.37: 6.0]$} & [15.3:109] & [0.32:0.71] & & & \\
\hline
\end{tabular}

a - Distribution used: exponential (exp), Dirac delta $(\delta)$, normal $(N)$, and lognormal $(\ln N)$.

$\mathrm{b}-$ Values in parantheses were held fixed

c $-95 \%$ confidence intervals are given in brackets for the normal and lognormal fits.

single-cycle, single-history yield experiment ( $\mathrm{SCSH})-$ mitigates complications tied to the accumulation of virus, and brings into focus the viral production of the cell culture as a series of snapshots over time, all sharing a common kinetic history.

Using the model in Equation (1), we performed fits to the SCSH data set [9] (Figure 5), allowing both the lengths of the latent and of the infectious periods to vary freely. To simulate the removal of the overlay medium at each sampling time, we reduced the virus concentration by a factor of $10^{3}$ (consideration of larger reductions showed a negligible influence on the fit). The first three data points were excluded from consideration while fitting since these points were likely due to the desorption of excess virus into the overlay [9], a process not accounted for in our model. The value of viral clearance was held fixed at $0.26 \mathrm{~h}^{-1}$, based on the results of an independent mock-infection experiment (Figure 7 of
[9]). Four model types were considered: exponential-, fixed-, normal- and lognormal-delay. The best-fit virus curves are shown in Figure 5. The fitted parameter, SSR and $\mathrm{AIC}_{\mathrm{c}}$ values for each distribution are reported in Table 3. As in SCG experiments, the normal-delay and lognormal-delay models provided adequate representations of the data, while the fixed-delay (DDE) and exponential-delay (ODE) models did not.

The unique attribute of the SCSH experiment is the view it provides of the viral production by the average infected cell over time. To investigate these kinetics, a transformation of the original data is required. Specifically, the experimental measure of accumulated virus $\Delta V$ over an interval $\Delta t$ can be converted to an average virus production rate for that time interval, i.e., the fraction of infectious cells multiplied by the (constant) viral production rate of a cell, $p I / N$. The theoretical relationship between these quantities is provided by 


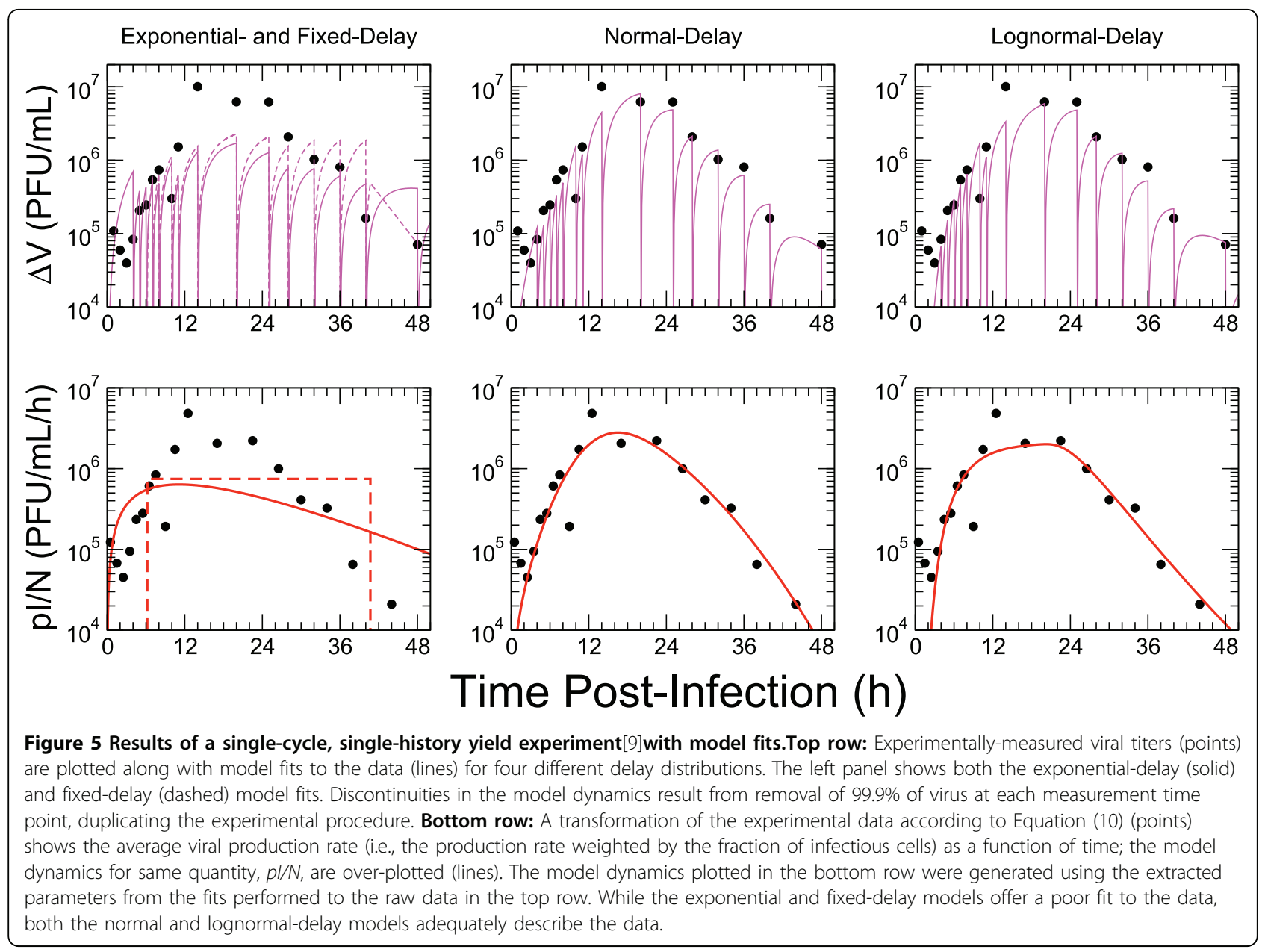

the basic equation for viral dynamics, Equation (1d). If we assume that the number of infectious cells, $I$, remains constant over the interval between samplings of the overlay, this equation can be solved to obtain accumulated virus as a function of average production rate and viral clearance, $c$ :

$$
\frac{\mathrm{d} V}{\mathrm{~d} t}+c V=\frac{p I}{N} \rightarrow V(t)=V(0) e^{-c t}+\frac{p I}{c N}\left(1-e^{-c t}\right) \rightarrow \Delta V=\frac{p I}{c N}\left(1-e^{-c \Delta t}\right),
$$

where we have used the fact that the virus concentration at the beginning of the time interval is zero. Rearranging this expression, the average viral production over an interval $\Delta t$ centered at time $t$ can then be written

$$
\frac{p I}{N}(t)=\frac{c \Delta V(t)}{1-e^{-c \Delta t}}
$$

The resulting transformed data is shown in Figure 5. Viewed in this way, one can explicitly see the growth of the infectious cell population, including a steep rise from $4 \mathrm{~h}$ to $12 \mathrm{~h}$ as cells transition from latent to infectious, and a long decline between $24 \mathrm{~h}$ and $48 \mathrm{~h}$ as infectious cells cease viral production. The model dynamics for $p I / N$, generated using parameters from the fit to the raw data, agree well with the transformed data, validating the use of Equation (1d) and the assumptions made in the above transformation.

While the normal-delay and lognormal-delay models both lead to an adequate description of the experimental data, a consideration of the fitted parameter values for the median infectious cell lifespan, $\tilde{t}_{I}$, reveals vastly different underlying dynamics. In the normal-delay case, the median infectious lifespan is predicted to be short $\left(\tilde{t}_{I} \approx 6.5 \mathrm{~h}\right)$ but the associated standard deviation is large. The long decay of infectious cells at late times is therefore explained by a broad distribution in the times spent by cells in the infectious state. Using the lognormal-delay model, however, the best fit is nearly that of a fixed-delay $\left(\sigma_{I} \ll 1\right)$, with a long median infectious lifespan $\left(\tilde{t}_{I} \approx 18 \mathrm{~h}\right)$. Under this assumption, the decay of infectious cells is thus completely determined by the long tail in the distribution of latently infected cell 
Table 3 Fits to the Gaush \& Smith (1968) single-cycle, single-history experiment (Figure 5) [9]

\begin{tabular}{|c|c|c|c|c|c|c|c|c|c|}
\hline Dist $^{a}$ & $p\left(\frac{\mathrm{PFU}}{\mathrm{h}}\right)$ & $\tau_{L}(\mathrm{~h})$ & $\sigma_{L}$ & $\tau_{l}(\mathrm{~h})$ & $\sigma_{l}$ & $\tilde{t}_{L}$ (h) & $\tilde{t}_{I}(\mathrm{~h})$ & SSR & $\mathrm{AIC}_{\mathrm{c}}$ \\
\hline$\overline{\exp }$ & $1.8 \times 10^{6}$ & 10.5 & - & 10.5 & - & 7.3 & 7.3 & 3.59 & -21.3 \\
\hline$\delta$ & $7.5 \times 10^{5}$ & 6.2 & - & 34.5 & - & 6.2 & 34.5 & $-^{\mathrm{b}}$ & - \\
\hline N & $\begin{array}{l}5.4 \times 10^{6} \\
{[1.4: 8.7]^{c}}\end{array}$ & $\begin{array}{l}12.4 \\
{[6.7: 14.3]}\end{array}$ & $\begin{array}{l}4.6 \mathrm{~h} \\
{[1.9: 5.8]}\end{array}$ & $\begin{array}{l}0.17 \\
{[0.002: 23]}\end{array}$ & $\begin{array}{l}9.7 \mathrm{~h} \\
{[4.3: 13.3]}\end{array}$ & 12.4 & 6.6 & 0.79 & -41.3 \\
\hline $\ln N$ & $\begin{array}{l}2.0 \times 10^{6} \\
{[1.0: 4.6]}\end{array}$ & $\begin{array}{l}8.5 \\
{[5.4: 10.8]}\end{array}$ & $\begin{array}{l}0.49 \\
{[0.24: 0.58]}\end{array}$ & $\begin{array}{l}18.2 \\
{[9.6: 25.6]}\end{array}$ & $\begin{array}{l}0 \\
{[0: 0.41]}\end{array}$ & 8.5 & 18.2 & 0.93 & -38.3 \\
\hline
\end{tabular}

a - Delay model used: exponential (exp), fixed $(\delta)$, normal $(N)$, and lognormal $(\ln N)$.

$\mathrm{b}$ - Using the fixed-delay model, virus titer is zero at all times prior to the appearance of infectious cells such that the SSR measured in log scale cannot be computed.

c $-95 \%$ confidence intervals are given in brackets for the normal and lognormal fits.

lifespans. With only a single data set, it is not possible to discriminate between these two extreme cases: short infectious lifespans on average with a broad distribution, versus a long average infectious lifespan with a narrow distribution of transitions. The results of SCG experiments, where viral titer is observed to grow linearly over 10 to $20 \mathrm{~h}$ (Figure 3), suggest that the former is unlikely since infectious cell death would lead to a turnover in the viral titer curve. It would be useful to duplicate this unique experiment in parallel with a typical SCG experiment such that these biologically distinct possibilities can be distinguished.

\section{Effect of delay assumptions in fitting clinical data}

We now consider the effect that the choice of delay distribution has on estimated parameter values when fitting a model to viral titer data from human patients experimentally infected with influenza. This type of data - two examples are shown in Figure 6, for others see [34] generally shows an exponential increase of virus followed by an exponential decrease after the peak, which occurs 1 to $2 \mathrm{~d}$ post-infection. An empirical function capturing these basic characteristics can be written as

$$
V(t)=\frac{2 V_{p}}{\exp \left[-\lambda_{g}\left(t-t_{p}\right)\right]+\exp \left[\lambda_{d}\left(t-t_{p}\right)\right]},
$$

where $\lambda_{g}$ and $\lambda_{d}$ are the exponential growth and decay rates, respectively. $V_{p}$ is the peak value of the viral titer, and $t_{p}$ is the time of viral titer peak. The exact peak values can be determined by differentiating Equation (11). Fits of this function to the experimental data, along with the calculated SSR, are shown in Figure 6. The simple functional form of the empirical model in Equation (11) points to a fundamental problem in fitting a dynamical model to clinical viral data: only four independent parameters can be reliably extracted from such data. A closely related problem is whether or not the parameters of a given model can be uniquely identified from a particular experimental measurement, the question of parameter identifiability [35-37]. It has been shown, for example, that the ODE model in Equation (3) is not identifiable when considering viral titer data alone [37]. Sparse experimental data, where the viral titer curve is not well-sampled, can introduce additional complications for model fitting. For example, in the influenza A/Texas/36/91 (H1N1) viral titer data [38] presented in Figure 6a, there is only a single data point (at $24 \mathrm{~h}$ ) from which the growth rate of viral titer, $\lambda_{g}$, can be determined.

To mitigate some of these problems, we fitted the four delay models (exponential-, fixed-, normal- and lognormal-delay) to the clinical data sets in Figure 6 under a number of constraints. We fixed the values of $\sigma_{L}$ and $\sigma_{I}$ to those obtained for SCSH experiment (Table 3) to allow for comparison of model systems with an equal number of parameters. Then, to allow for unique solutions in the fitting algorithm, we reduced the parameter space by fixing the product of the viral production and infection rates, $p \beta$, to the value of $1 \mathrm{~h}^{-2}$. This quantity is a measure of the infectivity of a virus-cell system and is related to the characteristic infecting time of the system, $t_{\text {inf }}=\sqrt{\frac{2}{p \beta}}$, which is time for a single infectious cell to cause the latent infection of one more in a completely susceptible cell population [33]. Under these constraints we were able to compare the effect of different delay assumptions on the fitted values of viral clearance, $c$, and production rate, $p$; median latent and infectious cell lifespans, $\tilde{t}_{L}$, and $\tilde{t}_{I}$, and the basic reproductive number $R_{0}$ (Table 4).

A comparison of the fitted parameter values shows a clear delineation between the results obtained under the assumption of exponentially-distributed delays and those of the other three models, which enforce longer delays for all cells. The median lifespan of a latently infected cell, $\tilde{t}_{L}$, is shorter under the exponential assumption (3.7 $\mathrm{h}$ vs. an average of $7.6 \mathrm{~h}$ for the A/Texas/36/91 $(\mathrm{H} 1 \mathrm{~N} 1)$ data set [38] and $6.4 \mathrm{~h}$ vs. an average of $9.4 \mathrm{~h}$ for A/Bethesda/1/85 (H3N2) [39]). The same is true for 

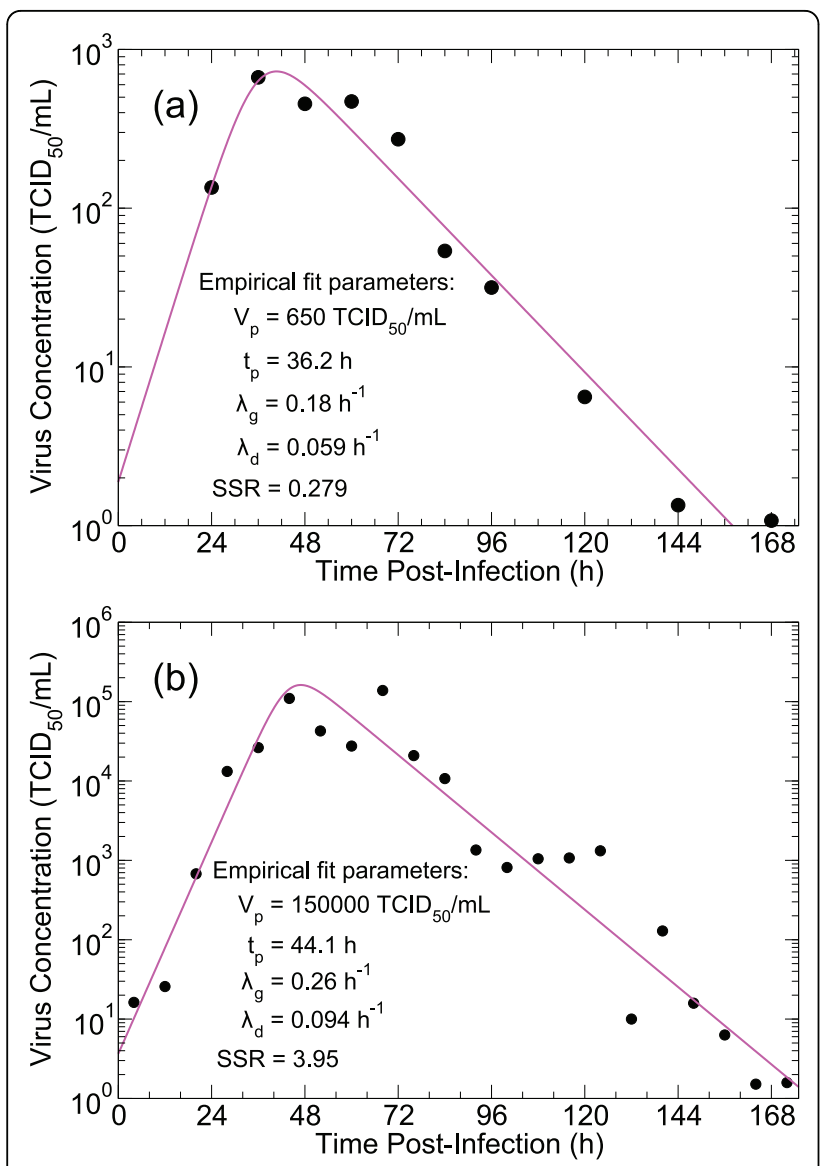

Figure 6 In vivo patient data. Viral titer over time for volunteer patients infected with (a) influenza A/Texas/36/91 (H1N1) [38], and (b) A/Bethesda/1/85 (H3N2) [39]. The solid curve is a fit of the empirical model in Equation (11) to each dataset. The fitted parameter values are given along with the SSR of the fit.

the median infectious lifetime, $\tilde{t}_{I}$. Extracted values for the viral production rate are larger for the exponentialdelay model, by a factor of $\sim 2$ in one data set and by an order of magnitude in the other. The estimated basic reproductive number is smaller for the exponentialdelay model, by a factor of $2-10$, than the other three delay types. The fitted value of the viral clearance, $c$, was independent of delay choice for one data set (and equal to the viral decay rate, $\lambda_{d}$ ), but differed for the exponential-delay model in the other.

\section{Conclusions}

Mathematical models of viral infections within a host or cell culture have helped shed light on several aspects of cell-virus interactions $[6,40,41]$. Most frequently, models have been used to extract values for the parameters controlling small-scale infection kinetics from experimental data $[4,42,43]$. This has allowed mathematical modeling to play an ever-growing role in virological and immunological studies, with an increasing number of publications in these fields incorporating some amount of modeling [44-46]. At present, however, modeling work often lags experimentation and primarily serves an explanatory role. It is desirable that models take a more predictive role in the future, where the simulation of viral infections may aid, for example, in the prediction of virulence for new strains or the kinetic mechanisms of untested antiviral therapies. Before models can take on a predictive role, however, the mathematical implementation of viral infection dynamics must be tested against a diverse set of experimental conditions to ensure that biological reality is faithfully represented.

Here, we have investigated the implementation of the progress of a cell through the states of infection: from latently infected to infectious to dead. We have focused on the characterization of two times: the time a cell is latently infected but not yet releasing virus, and the time a cell is infectious (releasing virus) before infection-induced death. We explored four different distributions for these state lifetimes: exponential, Dirac delta (fixed-delay), normal, and lognormal. The validity of each distribution was assessed by fitting the associated model to data from single-cycle growth (SCG) viral yield experiments. These experiments provide a unique view of the average dynamics of a single cell due to the synchronous infection of all cells. We have shown that ODE models which implement exponential delays and DDE models with fixed delays are unable to describe this experimental data, whereas normal-delay and lognormal-delay models both provide a good fit to the data. In addition to the classic SCG experiment, we have also considered delay dynamics for a "single-cycle, single-history" (SCSH) experiment [9], which reveals average cellular virus production as a function of time. We have shown that, like the SCG experiment, both normal- and lognormal-delay models provide an adequate description of the dynamics while exponentialand fixed-delay models do not. The origin of the inability of ODEs and DDEs to exhibit single-cycle dynamics can be seen quite clearly in the experimental data (Figures 3,4 , and 5): virus production begins only after a long delay following the infection of a cell, a feature which ODE models cannot replicate, and the transition of cells into and out of the infectious phase follows a smooth distribution which DDE models cannot reproduce.

The median values of the latent infection period, determined by fitting normal- and lognormal-delay models to the data from these SCG experiments, ranged from $8 \mathrm{~h}$ to $24 \mathrm{~h}$, which is significantly longer than the 4 to $6 \mathrm{~h}$ values typically quoted in the literature (e.g., [2]). There are a number of possible reasons for this discrepancy. First, the latent infection period in the model 
Table 4 Constrained model fits to the human influenza infection data in Figure 6

\begin{tabular}{|c|c|c|c|c|c|c|c|c|c|c|c|}
\hline \multicolumn{12}{|c|}{ A/Texas/36/91 (H1N1) [38] } \\
\hline Dist $^{a}$ & $c\left(h^{-1}\right)$ & $V_{0}^{\mathrm{b}}$ & $p^{b, c}$ & $\tau_{L}(\mathrm{~h})$ & $\sigma_{L}$ & $\tau_{l}(\mathrm{~h})$ & $\sigma_{l}$ & $\tilde{t}_{L}(\mathrm{~h})$ & $\tilde{t}_{I}(\mathbf{h})$ & $R_{0}$ & $S_{S R}^{d}$ \\
\hline $\exp$ & 0.19 & 0.022 & 280 & 5.4 & - & 16.9 & - & 3.7 & 11.7 & 89 & 1.00 \\
\hline$\delta$ & 0.059 & 2.85 & 62 & 10.2 & - & 23.0 & - & 10.2 & 23.0 & 390 & 1.00 \\
\hline$N$ & 0.059 & 0.013 & 100 & 1.8 & $(4.6 h)^{c}$ & 12.1 & $(9.7 \mathrm{~h})$ & 3.9 & 13.4 & 240 & 0.98 \\
\hline $\ln N$ & 0.059 & 0.72 & 99 & 8.7 & $(0.49)$ & 14.5 & (0) & 8.7 & 14.5 & 250 & 1.00 \\
\hline \multicolumn{12}{|c|}{ A/Bethesda/1/85 (H3N2) [39] } \\
\hline Dist & $c\left(h^{-1}\right)$ & $V_{0}$ & $p$ & $\tau_{L}(\mathrm{~h})$ & $\sigma_{L}$ & $\tau_{l}(\mathrm{~h})$ & $\sigma_{l}$ & $\tilde{t}_{L}(\mathrm{~h})$ & $\tilde{t}_{I}(\mathrm{~h})$ & $R_{0}$ & SSR \\
\hline $\exp$ & 0.11 & 7.0 & $1.3 \times 10^{5}$ & 9.0 & - & 2.2 & - & 6.4 & 1.5 & 20 & 0.91 \\
\hline$\delta$ & 0.10 & 28.4 & $7.7 \times 10^{3}$ & 9.9 & - & 31.5 & - & 9.9 & 31.5 & 320 & 0.88 \\
\hline$N$ & 0.10 & 10.6 & $1.2 \times 10^{4}$ & 9.6 & $(4.6 h)$ & 19.9 & $(9.7 \mathrm{~h})$ & 9.7 & 20.1 & 200 & 0.90 \\
\hline $\ln N$ & 0.10 & 11.8 & $8.0 \times 10^{3}$ & 8.5 & $(0.49)$ & 30.4 & (0) & 8.5 & 30.4 & 300 & 0.91 \\
\hline
\end{tabular}

a - Delay model used: exponential $(\exp )$, fixed $(\delta)$, normal $(N)$, and lognormal $(\ln N)$.

$\mathrm{b}-$ Units of $p$ are $[V] \cdot \mathrm{h}^{-1}$, where $[V]=\mathrm{TCID}_{50} / \mathrm{mL}$, the units of viral titer.

c - The value of $p \beta$ was held fixed at $1 \mathrm{~h}^{-2}$ for all fits; values in parentheses were also eld fixed.

$d-S^{\prime} R^{\prime}$ is the ratio of the SSR to that of the empirical fit.

includes both the typical eclipse period prior to virus production and any additional time required for viral release. Second, viral production in a culture is likely detectable much earlier. For example, under the assumption of normal delays the median length of the latent phase was found in the SCSH experiment to be $\tilde{t}_{L}=12.4 \mathrm{~h}$ with $\sigma_{L}=4.6 \mathrm{~h}$. Thus, $6 \%$ of cells $(\sim 600$, $000)$ had already begun releasing virus after only 6 h; using an alternative definition, the phase of latency could be declared over much earlier. Finally, the model describes a system where each cell releases no virus until the start of the infectious phase at which point virus is produced at a constant rate common to all cells. This is obviously a simplified version of reality. In fact, there is significant evidence from flow cytometry fluorescence experiments that different cells produce virus at different rates, perhaps over several orders of magnitude [47]; it is also likely that the rate of virus production varies over the course of its infectious lifespan.

The median infectious cell lifespan, determined in fits of the normal- and lognormal-delay models to the SCSH data, ranged from 6 to $18 \mathrm{~h}$, but the application of these two distributions types implied disparate dynamical scenarios. Under the assumption of normal delays, the infectious lifespan was small but the distribution was broad. In contrast, the lognormal assumption predicted nearly a fixed infectious lifetime for all cells and the observed slow decline of infections cells was completely ascribed to a long tail in the transition from the latent to infectious phase. Biologically, the infectious cell lifespan is variously characterized in the literature, as is influenzainduced cell death in general. When cell death is due to apoptosis, for example in MDCK cell cultures [48], the median time of cell death ranges from 12 to $48 \mathrm{~h}$ after infection, depending on the influenza strain subtype.
When virus-induced cell death is caused by necrosis, as in cultures of some lung and intestinal epithelial cells, this range increases significantly with cells living $2-3 \mathrm{~d}$ post-infection $[20,49,50]$. The death of cells during an infection in vivo is obviously much more difficult to measure. There is some evidence that influenza-induced cell death is caused by apoptosis [51-53] but details of the timing and strain dependence are unknown. Future elaboration of SCSH experiments, in concert with the type of analysis performed here, could aid in the quantitative characterization of virus-induced cell death.

Using the results from our analysis of in vitro experiments, we considered the effect of delay distribution choice when fitting viral titer data from in vivo infections (experiments performed on human volunteers). To allow for the identification of some kinetic parameters, we restricted the analysis by assuming a fixed value for the viral infectivity (defined as the product of the infection and production rates, $p \beta$ ) and used the values for the parameters $\sigma_{L}$ and $\sigma_{I}$ determined when fitting the in vitro data. Under these assumptions, we found a clear difference between the extracted parameter values for models enforcing a delay in transitions (fixed-, normaland lognormal-delay) and the ODE model with exponential transitions. Specifically the ODE predicted larger virus production rates, shorter latent and infectious phase lifespans, and a lower value of the basic reproductive number, $R_{0}$ (Table 4). Although this result depends on rather arbitrary assumptions - infectivity may vary by orders of magnitude and there is little reason to assume that parameter values determined in vitro should be the same in vivo - it demonstrates that the choice of delay distribution has a significant effect on the conclusions drawn from model-fitting of in vivo data. This analysis also suggests that while a fixed-delay 
model cannot reproduce the continuous dynamics of cell transitions observed in single-cycle experiments, it may be a reasonable substitute for the more complicated normal and lognormal models in some contexts.

Our analysis of the in vivo experiments demonstrates the difficulty in extracting reliable information from viral titer data alone. Consideration of the SSR values for the model fits to in vivo data (Table 4) shows that each model adequately describes the data, despite the arbitrary constraints imposed. This highlights both the weakness of using viral titer data for model selection and the difficulty in uniquely identifying parameters from such data, given a particular model. Precise statements about the parameters controlling an in vivo infection can only be made by either imposing constraints to reduce the considered parameter space (as we have done here), or by obtaining complementary data (for example information about infectious cell population dynamics). Collection of infected cell data over the course of an influenza infection has only recently been considered in animal models (e.g., [54]) and such data will likely be unobtainable for human infections. Full characterization of models using in vitro experiments will therefore remain an important direction of future research. The introduction of infection models with an explicit immune response and the parallel measurements of immune system quantities (which are easier to obtain than infected cell populations) is a promising direction not only for a more complete understanding of the influenza infection but also the complete parametrization of these models $[5,54,55]$ This will, of course, depend on the development of simple models for which the added complexity is warranted by the available data.

In the past ten years, tools for dynamical measurements of in vitro viral infections have improved quickly. The measurement of infection within individual cells by fluorescence microscopy has become routine [47]. The spatial spread of virus infections on cell culture can be viewed in real time, both at the level of cellular deformation and at the level of individual virus particles [56]. Individual virus particles have been tracked as they enter a cell, and repeated observations have allowed for a statistical characterization of the timing of events in the early stages of infection [16]. These detailed views of the influenza infection will be invaluable for the construction of the next generation of viral infection models. Models, in turn, will provide a cohesive picture of the overall infection process and, crucially, make connections between the known small-scale details of the virus-cell interaction and the infection at the level of the organism, where it is manifested as a disease.

\section{List of abbreviations}

ODE: Ordinary differential equation; DDE: Delay differential equation; SSR: Sum of squared residuals; AIC: Akaike information criterion; AIC $_{c}$ : Akaike information criterion, corrected for small samples; SCG: Single-cycle growth; MOI: Multiplicity of infection; SCSH: Single-cycle, single-history growth; MDCK: Madin Darby canine kidney; VU: Virus units

\section{Acknowledgements}

This study was supported by the Canadian Institutes for Health Research [funding reference number 86937] and by the Natural Sciences and Engineering Research Council of Canada (CAAB). The authors wish to thank Dr. Shingo Iwami for his careful reading of the manuscript and helpful comments.

This article has been published as part of BMC Public Health Volume 11 Supplement 1, 2011: Mathematical Modelling of Influenza. The full contents of the supplement are available online at http://www.biomedcentral.com/ $1471-2458 / 11$ ? issue $=$ S1.

\section{Authors' contributions}

$\mathrm{BH}$ conceived the study, participated in the design of the study, carried out the modeling, performed the analysis and drafted the manuscript. $C A A B$ conceived of the study, participated in its design and coordination, and helped to draft the manuscript. All authors read and approved the final manuscript.

\section{Competing interests}

The authors declare that they have no competing interests.

Published: 25 February 2011

\section{References}

1. Lenglet $A D$, Hernando V, Rodrigo P, Larrauri A, Donado JD, de Mateo S: Impact of flu on hospital admissions during 4 flu seasons in Spain, 2000-2004. BMC Public Health 2007, 7:197.

2. Möhler L, Flockerzi D, Sann H, Reichl U: Mathematical model of influenza A virus production in large-scale microcarrier culture. Biotechnol. Bioeng 2005, 90:46-58.

3. Beauchemin C, McSharry J, Drusano G, Nguyen J, Went G, Ribeiro R, Perelson $A$ : Modeling amantadine treatment of influenza $A$ virus in vitro. J. Theor. Biol 2008, 254:439-451.

4. Baccam P, Beauchemin C, Macken C, Hayden F, Perelson A: Kinetics of influenza A virus infection in humans. J. Virol 2006, 80:7590-7599.

5. Handel A, Longini I, Antia R: Towards a quantitative understanding of the within-host dynamics of influenza A infections. J. R. Soc. Interface 2010, 7:35-47

6. Ho D, Neumann A, Perelson A, Chen W, Leonard J, Markowitz M: Rapid turnover of plasma virions and CD4 lymphocytes in HIV-1 infection. Nature 1995, 373:123-126.

7. Smith AM, Ribeiro RM: Modeling the viral dynamics of influenza a virus infection. Crit. Rev. Immunol 2010, 30:291-298.

8. Dulbecco R, Vogt M: Plaque formation and isolation of pure lines with poliomyelitis viruses. J. Exp. Med 1954, 99:167-182.

9. Gaush C, Smith T: Replication and plaque assay of influenza virus in an established line of canine kidney cells. Appl. Environ. Microbiol 1968, 16:588-594.

10. Kermack W, McKendrick A: Contributions to the mathematical theory of epidemics, Part I. Proc. Roy. Soc. A 1927, 115:700-721.

11. Hethcote $H$, Tudor D: Integral equation models for endemic infectious diseases. J. Math. Biol 1980, 9:37-47.

12. Feng $Z, X u$ D, Zhao H: Epidemiological models with non-exponentially distributed disease stages and applications to disease control. Bull. Math. Biol 2007, 69:1511-1536.

13. Wearing $H J$, Rohani $P$, Keeling MJ: Appropriate models for the management of infectious diseases. PLOS Med 2005, 2:0621-0627.

14. Lloyd AL: Destabilization of epidemic models with the inclusion of realistic distributions of infectious periods. Proc. R. Soc. Lond., B, Biol. Sci 2001, 268:985-993.

15. Banks $H$, Bortz DM, Holte SE: Incorporation of variability into the modeling of viral delays in HIV infection dynamics. Math. Biosci 2003, 183:63-91.

16. Lakadamyali M, Rust MJ, Babcock HP, Zhuang X: Visualizing infection of individual influenza viruses. Proc. Natl. Acad. Sci. U.S.A 2003, 100:9280-9285

17. Martin $K$, Helenius $A$ : Nuclear transport of influenza virus ribonucleoproteins: The viral matrix protein (M1) promotes export and inhibits import. Cell 1991, 67:117-130. 
18. Nayak DP, Hui EKW, Barman S: Assembly and budding of influenza virus. Virus Res 2004, 106:147-165.

19. Takizawa T, Matsukawa S, Higuchi Y, Nakamura S, Nakanishi Y, Fukuda R: Induction of programmed cell death (apoptosis) by influenza virus infection in tissue culture cells. J. Gen. Virol 1993, 74:2347-2355.

20. Zhirnov $\mathrm{O}$, Klenk HD: Human influenza $A$ viruses are proteolytically activated and do not induce apoptosis in CACO-2 cells. Virology 2003, 313:198-212

21. Mohsin MA, Morris SJ, Smith H, Sweet C: Correlation between levels of apoptosis, levels of infection and haemagglutinin receptor binding interaction of various subtypes of influenza virus: Does the viral neuraminidase have a role in these associations. Virus Res 2002 85:123-131.

22. Lee HY, Topham DJ, Park SY, Hollenbaugh J, Treanor J, Mosmann TR, Jin X, Ward BM, Miao H, Holden-Wiltse J, Perelson AS, Zand M, Wu H: Simulation and prediction of the adaptive immune response to influenza $A$ virus infection. J. Virol 2009, 83:7151-7165.

23. Mittler JE, Sulzer B, Neumann AU, Perelson AS: Influence of delayed viral production on viral dynamics in HIV-1 infected patients. Math. Biosci 1998, 152:143-163.

24. Grossman Z, Feinberg M, Kuznetsov V, Dimitrov D, Paul W: HIV infection: How effective is drug combination treatment? Immunol. Today 1998, 19:528-532.

25. Lloyd AL: Dependence of viral parameter estimates on the assumed viral life cycle: Limitations of studies of viral load data. Proc. R. Soc. Lond., B, Biol. Sci 2001, 268:847-854.

26. Keele BF, Learn HLGH, Hraber P, Giorgi EE, Grayson T, Sun C, Chen $Y$, Yeh WW, Letvin NL, Mascola JR, Nabel GJ, Haynes BF, Bhattacharya T, Perelson AS, Korber BT, Hahn BH, Shaw GM: Low-dose rectal inoculation of rhesus macaques by SIVsmE660 or SIVmac251 recapitulates human mucosal infection by HIV-1. J. Exp. Med 2009, 206:51117-51134.

27. Zwart MP, Hemerik L, Cory JS, de Visser JAG, Bianchi FJ, Oers MMV, Vlak JM, Hoekstra RF, der Werf W: An experimental test of the independent action hypothesis in virus-insect pathosystems. Proc. R. Soc. Lond., B, Biol. Sci 2009, 276:2233-2242.

28. Hale B, Knebel A, Botting C, Galloway C, Precious B, Jackson D, Elliott R Randall R: CDK/ERK-mediated phosphorylation of the human influenza $A$ virus NS1 protein at threonine-215. Virology 2009, 383:6-11.

29. Min J, Krug R: The primary function of RNA binding by the influenza A virus NS1 protein in infected cells: Inhibiting the $2 \mathbb{2}-5 \rrbracket$ oligo(A) synthetase/RNase L pathway. Proc. Natl. Acad. Sci. U.S.A 2006, 103:7100-7105.

30. Nagai T, Moriguchi R, Suzuki Y, Tomimori T, Yamada H: Mode of action of the anti-influenza virus activity of plant flavonoid, 5,7,4区-trihydroxy-8methoxyflavone, from the roots of Scutellaria baicalensis. Antiviral Res 1995, 26:11-25

31. Kaverin $N$, Webster R: Impairment of multicycle influenza virus growth in Vero (WHO) cells by loss of trypsin activity. J. Virol 1995, 69:2700-2703.

32. Sugiura A, Kilbourne E: Genetic studies of influenza viruses II. Plaque formation by influenza viruses in a clone of a variant human heteroploid cell line. Virology 1965, 26:478.

33. Holder BP, Liao LE, Simon P, Boivin G, Beauchemin CA: Design considerations in building in silico equivalents of common experimental influenza virus assays. Autoimmunity 2011.

34. Carrat F, Vergu E, Ferguson NM, Lemaitre M, Cauchemez S, Leach S, Valleron AJ: Time lines of infection and disease in human influenza: A review of volunteer challenge studies. Am. J. Epidemiol 2008, $167: 775-785$

35. Xia X, Moog C: Identifiability of nonlinear systems with application to HIV/AIDS models. IEEE Trans. Automat. Contr 2003, 48:330-336.

36. Wu H, Zhu H, Miao H, Perelson A: Parameter identifiability and estimation of HIV/AIDS dynamic models. Bull. Math. Biol 2008, 70:785-799.

37. Miao H, Xia X, Perelson A, Wu H: On identifiability of nonlinear ODE models and applications in viral dynamics. SIAM Review 2011, To Appear

38. Barroso L, Treanor J, Gubareva L, Hayden FG: Efficacy and tolerability of the oral neuraminidase inhibitor peramivir in experimental human influenza: Randomized, controlled trials for prophylaxis and treatment. Antivir. Ther 2005, 10:901-910.

39. Bjornson A, Mellencamp M, Schiff G: Complement is activated in the upper respiratory tract during influenza virus infection. Am. Rev. Respir. Dis 1991, 143:1062-1066
40. Nowak M, Bonhoeffer S, Hill A, Bohme R, Thomas H, McDade H: Viral dynamics in hepatitis B infection. Proc. Natl. Acad. Sci. U.S.A 1996, 93:4398-4404.

41. Sidorenko $Y$, Reichl $U$ : Structured model of influenza virus replication in MDCK cells. Biotechnol. Bioeng 2004, 88:1-14.

42. Perelson A, Neumann A, Markowitz M, Leonard J, Ho D: HIV-1 dynamics in vivo: Virion clearance rate, infected cell life-span, and viral generation time. Science 1996, 271:1582-1586.

43. Ciupe SM, Ribeiro RM, Nelson PW, Dusheiko G, Perelson AS: The role of cells refractory to productive infection in acute hepatitis $B$ viral dynamics. Proc. Natl. Acad. Sci. U.S.A 2007, 104:5050-5055.

44. Bauer AL, Beauchemin CA, Perelson AS: Agent-based modeling of hostpathogen systems: The successes and challenges. Inf. Sci. (Ny) 2009, 179:1379-1389

45. Forrest S, Beauchemin CA: Computer immunology. Immunol. Rev 2007, 216:176-197.

46. Chavali AK, Gianchandani EP, Tung KS, Lawrence MB, Peirce SM, Papi JA: Characterizing emergent properties of immunological systems with multi-cellular rule-based computational modeling. Trends Immunol 2008, 29:589-599.

47. Sidorenko Y, Schulze-Horsel J, Voigt A, Reichl U, Kienle A: Stochastic population balance modeling of influenza virus replication in vaccine production processes. Chem. Eng. Sci 2008, 63:157-169.

48. Price G, Smith H, Sweet C: Differential induction of cytotoxicity and apoptosis by influenza virus strains of differing virulence. J. Gen. Virol 1997, 78:2821-2829.

49. Seo SH, Goloubeva O, Webby R, Webster RG: Characterization of a porcine lung epithelial cell line suitable for influenza virus studies. J. Virol 2001, 75:9517-9525.

50. Arndt U, Wennemuth G, Barth P, Nain M, Al-Abed Y, Meinhardt A, Gemsa D, Bacher $\mathrm{M}$ : Release of macrophage migration inhibitory factor and CXCL8/interleukin-8 from lung epithelial cells rendered necrotic by influenza A virus infection. J. Virol 2002, 76:9298-9306

51. Mori I, Komatsu T, Takeuchi K, Nakakuki K, Sudo M, Kimura Y: In vivo induction of apoptosis by influenza virus. J. Gen. Virol 1995, 76:2869-2873.

52. Ito T, Kobayashi Y, Morita T, Horimoto T, Kawaoka Y: Virulent influenza A viruses induce apoptosis in chickens. Virus Res 2002, 84:27-35.

53. Uiprasertkul M, Kitphati R, Puthavathana P, Kriwong R, Kongchanagul A, Ungchusak K, Angkasekwinai S, Chokephaibulkit K, Srisook K, Vanprapar N, Auewarakul P: Apoptosis and pathogenesis of avian influenza A (H5N1) virus in humans. 2007, 13:708-712.

54. Saenz RA, Quinlivan M, Elton D, MacRae S, Blunden AS, Mumford JA, Daly JM, Digard P, Cullinane A, Grenfell BT, McCauley JW, Wood JLN, Gog JR: Dynamics of infection and pathology in influenza. J. Virol 2010, 84:3974-3983.

55. Miao H, Hollenbaugh JA, Zand MS, Holden-Wiltse J, Mosmann TR, Perelson AS, Wu H, Topham DJ: Quantifying the early immune response and adaptive immune response kinetics in mice infected with influenza A virus. J. Virol 2010, 84:6687-6698.

56. Doceul V, Hollinshead M, van der Linden L, Smith G: Repulsion of superinfecting virions: A mechanism for rapid virus spread. Science 2010, 327:873-876

57. Bellman R, Åström K: On structural identifiability. Math Biosci 1970, 7:329-339

58. Cobelli C, III JJD: Parameter and structural identifiability concepts and ambiguities: a critical review and analysis. Am. J. Physiol. Regulatory Integrative Comp. Physiol 1980, 239:R7-R24.

59. Bellu G, Saccomania MP, Audoly S, D'Angió L: DAISY: A new software tool to test global identifiability of biological and physiological systems. Comput. Methods Programs Biomed 2007, 88:52-61.

60. Eaton JW: GNU Octave. 2010 [http://www.octave.org].

61. Burnham KP, Anderson DR: Multimodel inference: Understanding AIC and BIC in model selection. Sociol. Methods Res 2004, 33:261-304.

62. Efron B, Tibshirani R: Bootstrap methods for standard errors, confidence intervals, and other measures of statistical accuracy. Stat. Sci 1986, $1: 77-97$

doi:10.1186/1471-2458-11-S1-S10

Cite this article as: Holder and Beauchemin: Exploring the effect of biological delays in kinetic models of influenza within a host or cell culture. BMC Public Health 2011 11(Suppl 1):S10. 